\title{
Examination on risk prevention measures for environment and safety of food plants in Japan
}

\author{
Ze Liu ${ }^{1, \mathrm{a}}$ and Takehiro Tanaka ${ }^{1}$ \\ ${ }^{1}$ Toyo University, Japan
}

\begin{abstract}
Risk prevention measures to secure both pleasant environment and safety are indispensable particularly to food plants. In this study, current situations and causes of troubles, affairs and accidents which have taken place in Japanese food plants are reviewed. In addition, the introduction of human-based strategies integrated with IT/IoT technologies as well as sampling methods for airborne microbes which have been being highlighted these days is proposed as measures to protect environment and safety of food plants.
\end{abstract}

\section{Introduction}

Risk prevention measures to secure both pleasant environment and safety are indispensable particularly to food plants. In this study, current situations and causes of troubles, affairs and accidents which have taken place in Japanese food plants are reviewed. In the section on "accidents/affairs of food adulteration," the annual numbers of consultation cases and accidents/affairs in food plants are discussed. In the section about "food safety standards adopted by food manufacturers," measures for safety and security in food plants are reviewed and then the significance of conventional $5 \mathrm{~S}$ management to food plants and evolutionary $5 \mathrm{~S}$ as human-based analog measures are introduced. In addition, the implementation of human-based strategies integrated with IT/IoT technologies as well as sampling methods for airborne microbes which have been being highlighted these days is proposed as measures to protect environment and safety of food plants.

\section{Accidents/affairs of food adulteration}

\subsection{Annual numbers of consultation cases}

Figure 1 shows the annual numbers of inquiries concerning food adulteration addressed to the National Consumer Affairs Center of Japan.

The number drastically increased in fiscal 2013, due to a series of events about agricultural chemicals detected from frozen food products. Out of all 6,477 inquiries, 3,583 cases were related to chemical adulteration. Within a total of 1,852 cases reported about foreign matters found in foodstuffs including both restaurant and delivery foods, pest-related complaints had the highest number of cases at 345. They included "a body or leg of cockroach," "a fly or a maggot," and "insects other than cockroaches and flies."

Food production plants are supposed to be operated under the strict hygiene management. However, the fact that those insects were actually detected from food products means that a quality-control program or production facility is inappropriate in some plants more than likely. Furthermore, it is reported that not only a hair but also a tooth, filling, or nail was found in food. To prevent those problems, the observation of strict quality control is not an indispensable duty of a food plant alone, but of a whole food company.

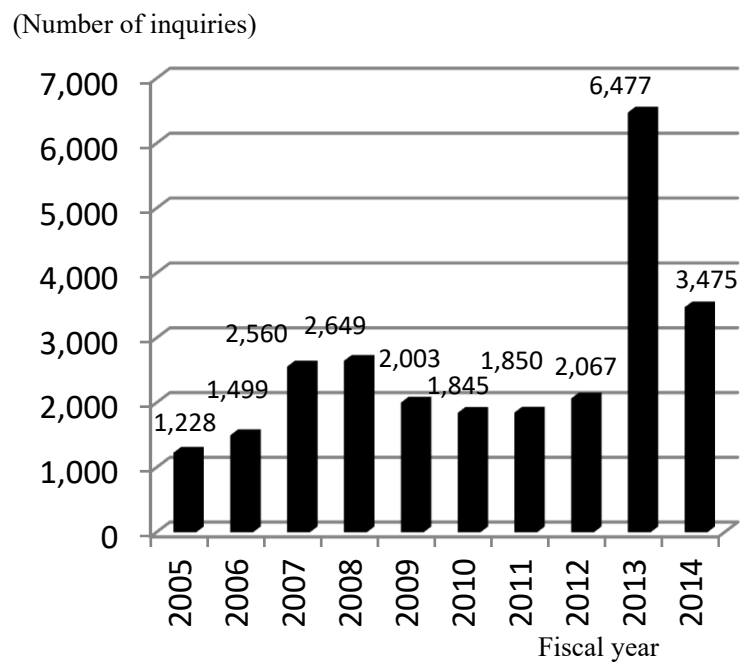

Figure 1. Annual numbers of inquiries about food adulteration

\footnotetext{
*Corresponding author: ahiya3506@yahoo.co.jp
} 


\subsection{Accidents/affairs in food plants}

Causes of faults of recalled food products are sorted out, as either "man-made or environmental," "intentional or accidental," and "by an insider or an outsider." Among man-made incidents, intentional misdeeds should be handled from a different viewpoint from those under the conventional safety management.

Because foreign matters are likely to cause ill affect to the human body, a food scandal may create distrust among consumers and dampen their willingness to buy the relevant products. Regardless of whether the offender is an insider or an outsider, if his/her deed is intentional, the foreign matter he/she puts in can be highly concentrated or massive in quantity. Thus, all companies are required to formulate their own preventive measures.

Damages to be recovered by the food company that has endangered food safety are direct losses and indirect losses. Direct losses include expenses for the recall and disposal of defective products and also compensation to the sufferers. Indirect losses are caused by the suspension of business transaction and the diminution of brand value. In general, no matter whether intentional or accidental, when a company gives rise to a scandal, the diminution of brand value as one of indirect losses seems to suffer the severest blow.

\section{Food safety standards adopted by food manufacturers}

Food Safety Management System (FSMS) is one of the approaches to the realization of food safety. FSMS is a general term for such programs as to properly control causal factors which threaten food safety. There are various FSMS standards and certificates.

\section{Measures for safety and security in food plants}

Food plant facilities are operated under either a closed system or an open system. Facilities under the closed system get rid of risk factors from the external environment. Giving priority to hygiene, they are automated with less human intervention. Their facilities, machines and systems are mostly maintained in a sanitary condition. Beverage manufacturing is one example. Meanwhile, delicatessen and confectionery are prepared through human intervention under the open system, where proper hygiene depend heavily on the practice of employees. Because the operation is conducted openly, the sanitary work environment is one of the most crucial factors.

To produce safe foods, "food safety" and "food defense" principles should be taken into consideration. "Food safety" refers to proper hygiene management to prevent food-borne illness. Specifically, such measures are taken as to prevent foods from decaying and foreign matters including pests and metallic pieces from getting in. In this system, the efficiency of an air shower room and the effectiveness of special work clothes are rigorously inspected. "Food defense" on the other hand is the prevention or protection of food products from intentional contamination or adulteration, by taking appropriate measures to hinder the occurrence of incidents like the past cases of pesticide contamination which took place in the frozen food plant. Specific preventive measures include the appropriate education given to the employees, and the installation of security cameras to monitor and check a trespasser and a hazardous material coming from outside.

If once adulteration (food terrorism) takes place at a food plant, it does not merely injure consumers' health but also disgraces the corporate brand and loses trust of business partners and customers, which eventually discourages customers from purchasing the products and begets groundless rumors. It also entails enormous costs such as the cost of food recalls. Thus the company consequently incurs substantial damage. For this reason, measures on the "food safety" and "food defense" principles have come to be regarded as important.

\section{Human-based analog measures}

\subsection{Significance of $5 \mathrm{~S}$ management to food plants}

In Japan, 5S management has been esteemed as human-based analog measures for food plants.

The 5S management advocates following five practices which commonly have the first letter of the word starting with "s" in Japanese. They are seiri (orderliness), seiton (tidiness), seiso (cleanness), seiketsu (hygiene), and sitsuke (discipline).

The present $5 \mathrm{~S}$ management in the food plants requires orderliness (seiri), tidiness (seiton), and cleanness (seiso), with discipline (sitsuke) as the foundation for achieving a main goal of good hygiene (seiketsu).

Hygiene (seiketsu) refers to the environment where foods are not ill affected. That is, contamination by microorganisms and chemicals as well as adulteration of foreign matters will never take place in this environment. To put it more concretely, efforts are devoted to create "the environment where foreign matters are hardly mixed in," to make "whatever's missing noticeable," and to improve "a sense of awareness of abnormal conditions." Orderliness (seiri), tidiness (seiton), and cleanness (seiso) are maintained to satisfy the required hygiene (seiketsu) as a goal.

\subsection{Evolutionary 5S}

In order to make the $5 \mathrm{~S}$ slogans more functional for the food industry, various approaches are currently tried on the daily basis, whereas there are some movements growing to reconsider the concept and effectiveness of the $5 \mathrm{~S}$ management.

One example is the promotion of the $7 \mathrm{~S}$ program for food sanitation. In this concept, it is suggested that washing (senjo) and sterilization (sakkin), which are 
currently represented by cleanness (seiso), be made independent and their purposes and methods be clarified. It advocates that these three $\mathrm{Ss}$ should be treated separately because they are important as countermeasures against microorganisms in any food plant. Thus, this particular program aims to achieve the prevention of not only adulteration but also contamination by microorganisms with utmost certainty.

\subsection{Bacteriological inspection}

As stated in Section 5.2, bacteriological inspections have been being introduced to food plants nowadays. Sampling methods for airborne microbes are considered quite important.

Activities of bacteriological inspections are grouped according to their purposes: "control inspection of raw materials and finished products," "inspection of manufacturing environment," "investigation of the cause of trouble," and "data collection related to product development."

The sampling methods of airborne microbes are either passive or active monitoring; passive air sampling devices such as settle plates and active air sampling devices such as impact, liquid impingement, and filtration. The extraction methods of microbes collected include incubation, luminescence and ATP analyses.

In the passive monitoring method, agar media are placed on desks and floors to catch falling microbiological particles. Microbiological colonies are cultivated on the agar media and counted in the incubation analysis. This method is often used to comprehend the surface contamination by airborne bacteria. The falling speed of microbiological particles to be collected depends on the particle size and airborne particles are subjected to airflow.

Most samplers widely available for the active monitoring let airborne microbes collide with agar media also in the incubation method. Impingers used in the liquid impingement method are sold commercially for microbes. To extract microbes collected in the liquid, they are inoculated into agar media or stained with fluorescent dye.

In the filtration method, microbes are captured on a membrane filter or gelatin filter.

Among the extraction measures, the incubation method requires several days for microbes to develop. Therefore, as practical prompt measures, microbes collected are stained with fluorescent dye and counted through a microscope, or ATP of microbes are measured.

The ISO16000 (Indoor Air) series related to indoor environment ${ }^{[4-7]}$ define standards for assessing the concentration of airborne fungi. Furthermore, the calculation of spores through a microscope has been recently standardized under ISO 16000-20.

In the conventional method, airborne microbes are collected and incubated before counting the number of colonies, where it usually takes several days. Since fluorescent staining was researched and developed nearly 20 years ago, the bacteriological inspection methods in the food industry have been developing.

\section{Measures on the basis of digital technologies (IT/loT)}

One of the noticeable things in terms of food defense is to check the behavior of operators by strengthening internal controls. Human-based analog measures discussed in Section 5 aim to prevent food adulteration in view of human nature as inherently good. The food defense principle introduced in this section makes the most of the digital (IT/IoT) technologies to check the behavior of operators in view of human nature as evil.

In pushing forward with a food defense program, an in-house security policy needs to be formulated. To start with, a managerial security level should be defined for each section within the plant. Then, whether a given area in the production/storage sections is where an imprudent action can be easily committed or not is determined. For each area, check points are set up in reference to the layout, zoning and flow lines of the plant. The recent trend in digital (IT/IoT) technologies to facilitate the food defense management is examined below.

\subsection{Surveillance system of production lines}

Among all security facilities, a system of security cameras is relatively reasonable and practical for food manufactures to install in a short time.

Security cameras have been fixed exclusively at a doorway of a plant and an entrance hall of an office. In recent years, those cameras are placed on the food production line for quality assurance. Under this system, video data of entry/exit and behavior of employees are recorded. In this light, highly advanced video technology with higher-resolution and longer-time recording capacities is admired.

\subsection{Hands-free access control system}

The hands-free access control system identifies an individual who carries a non-directional IC tag with a 3D antenna. Unlike the contactless IC card system, which requires a user to put the card near to the reader, an IC tag is wearable even though a sanitary workwear has no outside pocket. Thus, this system is operable together with a sanitary workwear and an air-blow apparatus. This system is very convenient in a food plant where dust and bacteria are thoroughly controlled through the practices of hand-washing and sterilization.

\section{Summary}

In this study, current situations and causes of troubles, affairs and accidents which have taken place in Japanese food plants were reviewed. In the section on "accidents/affairs of food adulteration," the annual numbers of consultation cases and accidents/affairs in food plants were discussed. In the section about "food safety standards adopted by food manufacturers," measures for safety and security in food plants were reviewed and then the significance of conventional $5 \mathrm{~S}$ management to food plants and evolutionary $5 \mathrm{~S}$ as

*Corresponding author: ahiya3506@yahoo.co.jp 
human-based analog measures were introduced. In addition, the implementation of human-based strategies integrated with IT/IoT technologies as well as sampling methods for airborne microbes which have been being highlighted these days was proposed as measures to protect environment and safety of food plants.

Particularly in a food value chain, great caution must be observed from top to bottom: farm $\rightarrow$ manufacturing plant $\rightarrow$ storage $\rightarrow$ distribution facility $\rightarrow$ retail shelf. In this regard, the use of digital (IT/IoT) technologies is indispensable. Meanwhile, analog (human-based) countermeasures are also inevitable to deal with disgraceful affairs which may take place in the food plants, grievances/objections among employees against work environment, whistle-blowing, and complaints from customers.

\section{References}

1. National Consumer Affairs Center of Japan. (2018, January 11). Retrieved from http://www.kokusen.go.jp/

2. https://www.iso.org/home.html

3. International Organization for Standard (2008): ISO 16000-16 Indoor air -Part 16 Detection and enumeration of moulds -Sampling by filtration

4. International Organization for Standard (2011): ISO 16000-18 Indoor air -Part 18 Detection and enumeration of moulds -Sampling by impaction

5. International Organization for Standard (2008): ISO 16000-17 Indoor air -Part 17 Detection and enumeration of moulds -Culture-based method

6. International Organization for Standard (2014)

7. T. Fujii, et al. Plant Security Management in Food Defense. Retrieved January 11, 2018 from Hitachi Review Web site: http://www.hitachihyoron.com/jp/pdf/2014/12/. 